\title{
BMJ Open Optimising acute stroke care organisation: a simulation study to assess the potential to increase intravenous thrombolysis rates and patient gains
}

\author{
Maarten M H Lahr (D) , Durk-Jouke van der Zee, ${ }^{2}$ Gert-Jan Luijckx, ${ }^{3}$ \\ Erik Buskens ${ }^{1,3}$
}

To cite: Lahr MMH, van der Zee D-J, Luijckx G-J, et al. Optimising acute stroke care organisation: a simulation study to assess the potential to increase intravenous thrombolysis rates and patient gains. BMJ Open 2020;10:e032780. doi:10.1136/ bmjopen-2019-032780

- Prepublication history and additional material for this paper are available online. To view these files, please visit the journal online (http://dx.doi. org/10.1136/bmjopen-2019032780).

Received 05 July 2019 Revised 04 December 2019 Accepted 05 December 2019

Check for updates

(c) Author(s) (or their employer(s)) 2020. Re-use permitted under CC BY. Published by BMJ.

${ }^{1}$ Department of Epidemiology, University of Groningen, University Medical Centre Groningen, Groningen, The Netherlands

${ }^{2}$ Department of Operations, Faculty of Economics and Business, University of Groningen, Groningen, The Netherlands

${ }^{3}$ Department of Neurology, University of Groningen, University Medical Centre Groningen, Groningen, The Netherlands

Correspondence to Dr Maarten M H Lahr; m.m.h.lahr@umcg.nl

\section{ABSTRACT}

Objectives To assess potential increases in intravenous thrombolysis (IVT) rates given particular interventions in the stroke care pathway.

Design Simulation modelling was used to compare the performance of the current pathway, best practices based on literature review and an optimised model.

Setting Four hospitals located in the North of the Netherlands, as part of a centralised organisational model. Participants Ischaemic stroke patients prospectively ascertained from February to August 2010.

Intervention The interventions investigated included efforts aimed at patient response and mode of referral, prehospital triage and intrahospital delays.

Primary and secondary outcome measures The primary outcome measure was thrombolysis utilisation. Secondary measures were onset-treatment time (0TT) and the proportion of patients with excellent functional outcome (modified Rankin scale (mRS) $0-1$ ) at 90 days. Results of 280 patients with ischaemic stroke, 125 (44.6\%) arrived at the hospital within 4.5 hours, and $61(21.8 \%)$ received IVT. The largest improvements in IVT treatment rates, 0TT and the proportion of patients with $\mathrm{mRS}$ scores of $0-1$ can be expected when patient response is limited to $15 \mathrm{~min}$ (IVT rate $+5.8 \%$; $0 \mathrm{TT}-6 \mathrm{~min}$; excellent $\mathrm{mRS}$ scores $+0.2 \%$ ), door-to-needle time to $20 \mathrm{~min}$ (IVT rate $+4.8 \%$; $0 \mathrm{TT}-28 \mathrm{~min}$; excellent $\mathrm{mRS}$ scores $+3.2 \%$ ) and 911 calls are increased to $60 \%$ (IVT rate $+2.9 \%$; 0TT $-2 \mathrm{~min}$; excellent $\mathrm{mRS}$ scores $+0.2 \%$ ). The combined implementation of all potential best practices could increase IVT rates by $19.7 \%$ and reduce $0 T$ by $56 \mathrm{~min}$.

Conclusions Improving IVT rates to well above 30\% appears possible if all known best practices are implemented.

\section{INTRODUCTION}

Intravenous thrombolysis (IVT) is an effective therapy for acute ischaemic stroke up to 4.5 hours after the onset of symptoms. ${ }^{12}$ This therapy is substantially underused, however, with $8 \%-10 \%$ of all stroke patients worldwide

\section{Strengths and limitations of the study}

- The simulation modelling study included a comprehensive collection of patient level data from both the prehospital and intrahospital acute stroke pathway.

- A simulation-based approach, as presented in this paper, can be instrumental in facilitating a broad overview of the set-up and performance of stroke pathways.

- Effects of capacity constraints on patients' waiting for care services are not explicitly modelled given their high priorities, allowing them to queue jump.

- Costs items associated with the proposed interventions could not be collected and controlled for.

- Estimations of time intervals used for model building might have changed over time.

currently receiving IVT. ${ }^{3}{ }^{4}$ In contrast, treatment rates of up to $35 \%$ have been achieved in optimised settings. ${ }^{56}$ The organisation of stroke care is an important factor in realising timely hospital arrival and treatment. ${ }^{78}$ The centralisation of care at designated stroke centres has been demonstrated to increase the proportion of patients arriving at the hospital in time for acute treatment. ${ }^{9-11}$ Given the substantial decrease in the benefit of treatment with increasing time delays, further efforts to expedite hospital arrival and subsequent treatment remain of crucial importance. $^{12} 13$

Various studies have investigated factors associated with efficiency in each part of the acute stroke pathway. Although it has been generally established that delay on the part of patients and/or bystanders is a primary factor in delaying hospital arrival, ${ }^{14}$ interventions aimed at improving optimal response by calling 911 immediately have exhibited varying success, and many lack sustained 
implementation ${ }^{15}$ (as is the case in other domains of medicine as well). ${ }^{16}$ Ambulance transportation to hospitals that offer acute treatment is associated with shorter onset-to-door times, reductions in intrahospital delays and increases in treatment rates. ${ }^{15} 1718$ The provision of acute treatment by a mobile stroke unit (MSU) before hospital arrival has been identified as a promising method for reducing time to IVT. ${ }^{19}$ Another widely studied topic concerns reducing the time between hospital arrival and treatment (door-to-needle (DTN) time), with reported DTN times as low as 20 min. ${ }^{20}$

The aforementioned studies have demonstrated clear benefits in terms of time saved. They nevertheless lack a broader overview of pathway set-up and its performance. Instead of addressing the solution space as a whole, they target isolated elements of the stroke pathway. Thelack of a broader overview is due in part to the predominant use of randomised controlled trials (RCT) as the main research vehicle. Given the effort involved in their setup, RCT studies focus predominantly on separate and singular elements of pathway performance. They may therefore be less suitable for investigating complex care systems (eg, acute stroke treatment). In particular, timely hospital arrival and the treatment of acute stroke patients relies on a series of intertwined activities concerning patient diagnostics and transportation.

One potential alternative methodology is simulation modelling. Proceeding from a detailed description of both prehospital and intrahospital time delays and diagnostics, an accurate representation of pathway performance can be developed in silico, including the validation of IVT rates, time to treatment, patient outcomes (as measured by the modified Rankin Scale (mRS)) and other clinically relevant outcome measures. ${ }^{21} 22$ This approach would allow the examination of all time delays and diagnostic steps, thereby providing clinicians and policymakers with an accurate overview of obstacles currently existing within care pathways. In addition, scenarios for hypothetical approaches to improvement based on clinical guidelines, literature observations and/or expert opinion can be modelled and studied for their cumulative effects on relevant clinical outcome measures. ${ }^{23} 24$

The aims of this simulation-modelling study were (1) to estimate the cumulative potential for improving IVT utilisation by implementing best practices on the organisation of the stroke pathway and, subsequently, (2) to explore areas in which further improvement is needed in order to achieve a fully optimised setting, in addition to identifying obstacles to such optimisation.

\section{METHODS}

This article is based on a 6-month, prospective, multicentre study performed in a centralised organisational stroke-care setting in the north of the Netherlands from February through July 2010. ${ }^{9}$ Patient-level data were collected on time delays and diagnostics, thereby providing detailed insight into patient flow and potential

\begin{tabular}{lc}
\hline $\begin{array}{l}\text { Table } 1 \text { Descriptive statistics of activity durations and } \\
\text { diagnostics }\end{array}$ \\
\hline Number of patients & 280 \\
\hline Age in years (SD) & $10(14)$ \\
\hline Male (\%) & $156(56)$ \\
\hline Patient responsiveness & \\
\hline $\begin{array}{l}\text { Time from symptom onset to call for help, } \\
\text { valid cases (\%) }\end{array}$ & $41(5-130)$ \\
\hline Median, min (IQR) & $129(46)$ \\
\hline Mode of referral (\%) & $84(30)$ \\
\hline General practitioner & $60(21)$ \\
\hline 911 & $7(3)$ \\
\hline Self-referral & $213(76)$ \\
\hline In-hospital patients & $9(7-12)$ \\
\hline Pathway set-up & $20(15-25)$ \\
\hline Transported by EMS (\%) & $17(9-22)$ \\
\hline Median response time, min (IQR) & $2(0-15)$ \\
\hline Median on scene time, min (IQR) & $12(6-15)$ \\
\hline Median transportation time, min (IQR) \\
\hline $\begin{array}{l}\text { Median time from hospital arrival to } \\
\text { neurological examination, min (range) }\end{array}$ \\
$\begin{array}{l}\text { Median time from hospital arrival to CT } \\
\text { examination min (IQR) }\end{array}$ \\
$\begin{array}{l}\text { Median time from hospital arrival to } \\
\text { laboratory examination, min (IQR) }\end{array}$ \\
\hline Median door to IVT time, min (IQR) \\
\hline
\end{tabular}

EMS, emergency medical services; IVT, intravenous thrombolysis.

obstacles in both the prehospital and intrahospital pathways (table 1). A schematic overview of patient flow and the steps included in the analyses is presented in figure 1.

\section{Setting and participants}

The centralised organisational setting consisted of four hospitals in the northern region of the Netherlands, with IVT being administered only in the University Medical Center Groningen (UMCG). Together with the other three community hospitals, general practitioner (GP) offices and emergency medical services (EMS), arrangements were made to transport presumed stroke victims immediately to the UMCG, thus bypassing community hospitals that might have been located closer to the patient's location. The international protocol for IVT (adjusted European Cooperative Acute Stroke Study (ECASS) III $^{25}$ and the regional protocol for prehospital management were followed. The region addressed in this study comprises approximately 580000 inhabitants, with a population density of 250 inhabitants $/ \mathrm{km}^{2}$. The study population consisted of ischaemic stroke patients admitted to all four hospitals between February and August 2010. Case ascertainment was confirmed by the 
PRE-HOSPITAL

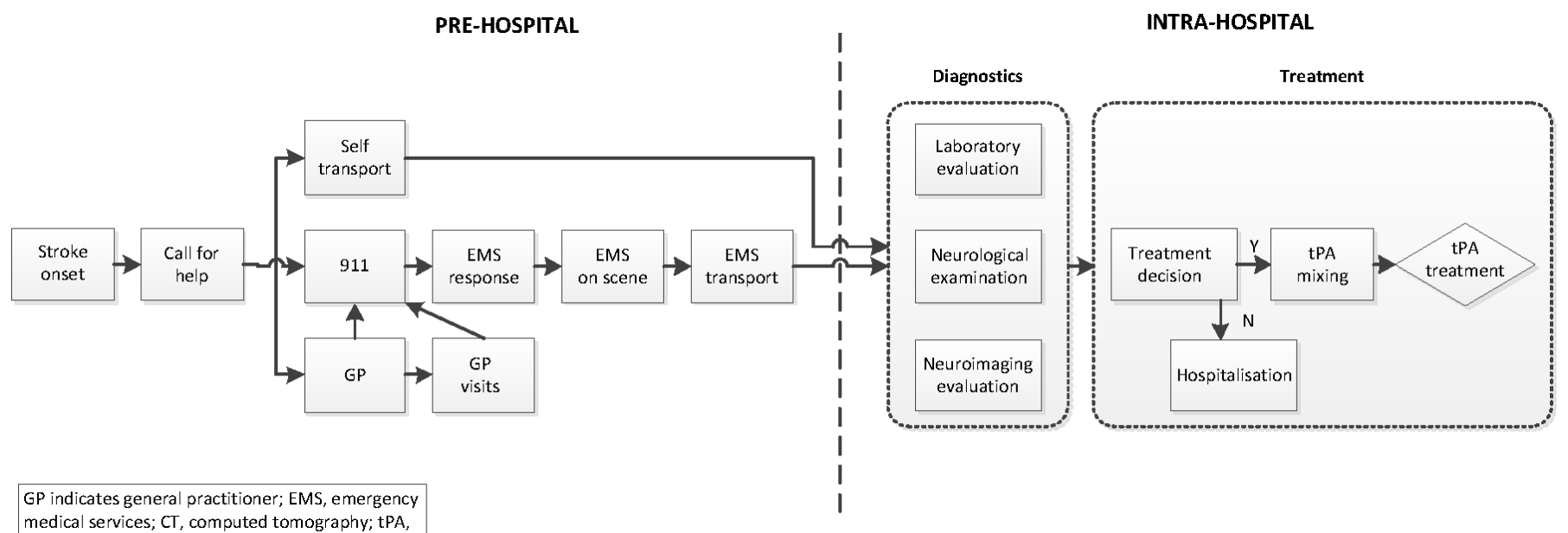

tissue plasminogen activator; $\mathrm{Y}=\mathrm{yes} ; \mathrm{N}=\mathrm{no}$

Figure 1 Acute stroke pathway: description of activities. EMS, emergency medical services; GP, general practitioner; N, no; tPA, tissue plasminogen activator; $Y$, yes.

final hospital discharge diagnosis of ischaemic stroke, thereby excluding stroke mimics.

The data collected included information on time delays and diagnostics along the entire stroke chain. Stroke care pathways can be described in several distinct phases: hyperacute (emergency), acute and rehabilitation. Within this study we focused on the hyperacute phase, ranging from symptom onset until acute treatment with IVT. Time delays included the time from symptom onset to call for help, delay at first response (GP or EMS services), EMS transport times and intrahospital diagnostics, which included time from hospital arrival to neurological examination, CT scan, laboratory testing and IVT.

\section{Simulation model}

The current study was performed using a previously validated simulation model. ${ }^{24}$ More detailed information on simulation modelling methodology, input parameters, model and model data used may be found in a online supplementary file. This model was populated with patient-level data from a previous observational study. ${ }^{9}$ The model was validated by comparing IVT treatment percentages and onset-treatment time (OTT) to those reported in the observational study. The next step consisted of developing scenarios in which alternative pathway set-ups, associated time delays and diagnostics were imputed based on literature observations, clinical guidelines and expert opinion. Using the simulation model, hypothetical patients were passed through the system, estimating the impact of intervening at various points in the acute stroke pathway. Interventions were modelled by changing the underlying statistical distributions to redistribute patients across time delays and diagnostics. ${ }^{26}$

\section{Scenarios}

We used simulation modelling to investigate the effects of changing pathway set-ups, based on three models: (1) a baseline model of acute stroke care; (2) a model reflecting best practices, based on a review of the current literature, clinical guidelines and expert opinion; and (3) an optimised model. Interventions were selected according to the obstacles identified in the current centralised organisational model. Obstacles within the study setting included delayed emergency response by patients following symptom onset, mode of referral (GP or 911), time spent on the scene by ambulance personnel and intrahospital delays. The simulation model was used to perform hypothetical interventions in the pathway to calculate clinically relevant outcomes. ${ }^{24}$ The outcomes were compared with the baseline performance of the current system to estimate the potential for improvement, to optimise system performance, and to identify obstacles that have yet to be overcome.

\section{Baseline}

The baseline model describes the performance of the centralised organisational model for acute stroke care, as described in the previous observational study. ${ }^{9} \mathrm{~A}$ description of time delays and diagnostics along each step of the pathway is provided in table 1 .

\section{Best practice}

The scenarios and input parameters that were investigated are described in table 2.

Patient responsiveness: We estimated the relative impact of reducing the time between stroke onset and call for help by patients, their families and/or bystanders by adjusting the distribution of their response times by a factor equal to the quotient of the respective median response times reported for best practices $(15-30 \mathrm{~min})^{27} 28$ and the baseline scenario (41 min).

Mode of referral: We modelled a scenario in which patients, their families and/or bystanders predominantly (60\%) chose 911 as the mode of entry. ${ }^{29} 30$

Time spent on the scene by ambulance personnel: In this scenario, we modelled the time spent on the scene by ambulance personnel following a 911 call by imposing an upper boundary on on-scene delay. Based on the guidelines of the American Stroke Association, the time spent on the 
Table 2 Overview of scenarios and input parameters

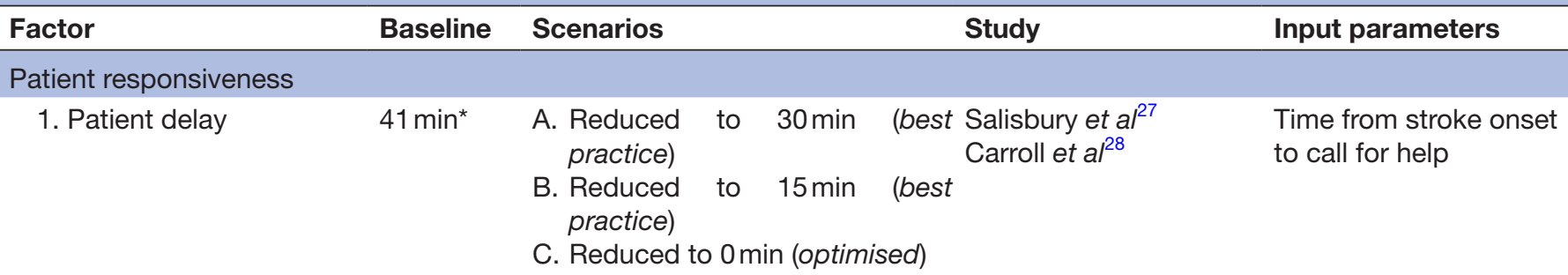

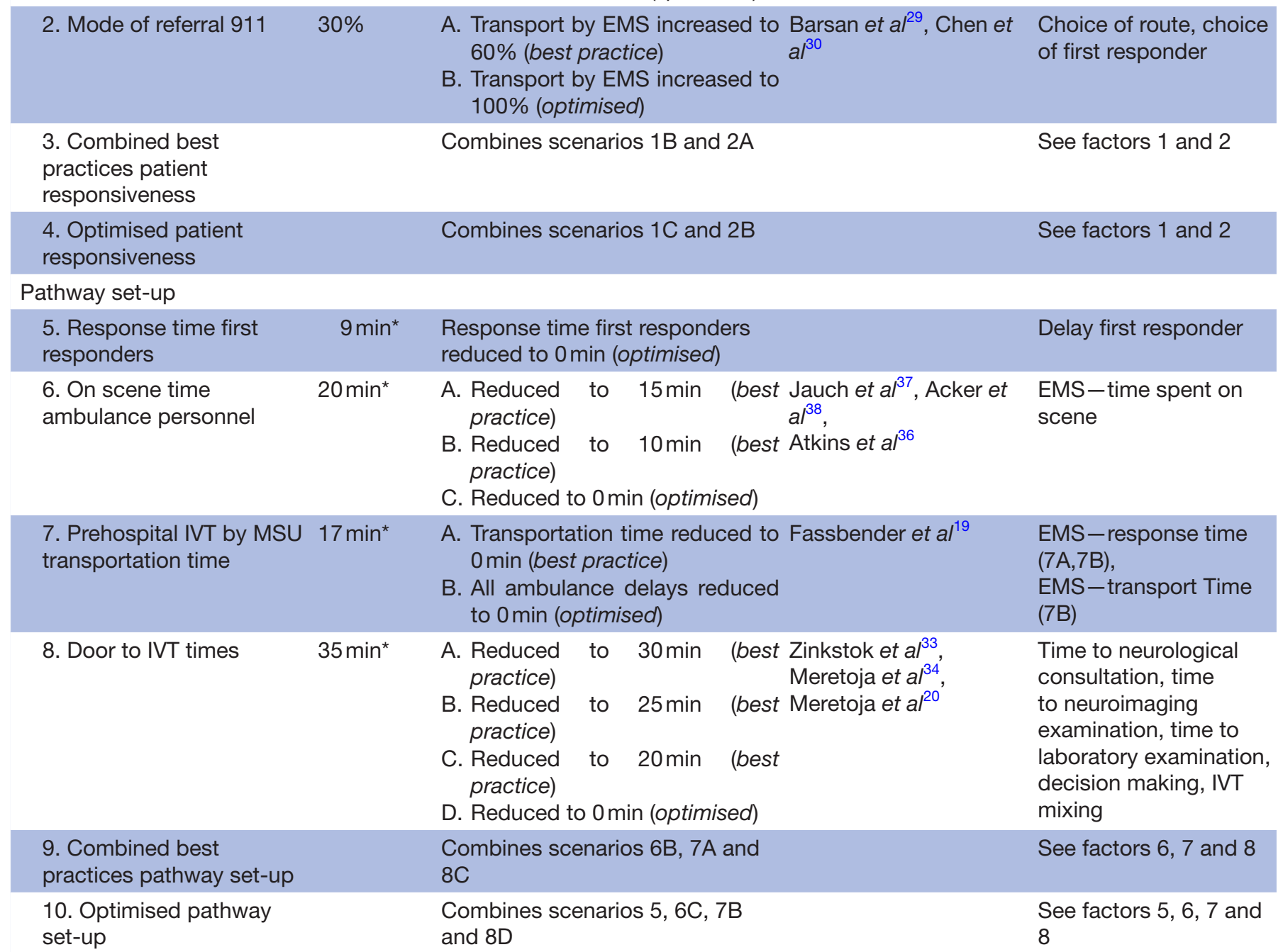

Patient responsiveness and pathway set-up

$\begin{array}{lll}\text { 11. Combined best } & \text { Combines scenarios } 3 \text { and } 9 & \text { See factors } 3 \text { and } 9\end{array}$

practices patient

responsiveness and

pathway set-up

12. Optimised patient

Combines scenarios 4 and 10

See factors 4 and 10 responsiveness and pathway set-up

*Median.

EMS, emergency medical services; IVT, intravenous thrombolysis; MSU, mobile stroke unit.

scene should be no more than $15 \mathrm{~min} .{ }^{31}$ We also modelled the implementation of a 'scoop and run protocol', which includes prompt transport $(\leq 10 \mathrm{~min})$ with initial management efforts, while postponing elaborate triage.
Prehospital treatment by MSU: In this scenario, we modelled prehospital IVT provided by a MSU by adjusting only the transport time of the ambulance. Assuming that the MSU would be stationed at the hospital, we considered only 
the transportation time from the hospital to the patient scene.

Intrahospital processes: Intrahospital delays comprise all activities performed between arrival at the hospital until the start of IVT (DTN time; see figure 1). Based on the guidelines of the American Stroke Association, DTN time should be no more than 60 min. ${ }^{32}$ Evidence from clinical practice suggests that DTN times within a range of 20-30 min are attainable. ${ }^{2033} 34$

\section{Combined best-practice scenarios}

Three scenarios were performed in which best practices were combined for patient responsiveness and pathway set-up. Patient responsiveness was modelled by combining patient response with the mode of referral following stroke onset. Pathway set-up was modelled by combining prehospital and intrahospital best practices. A third scenario combines best practices for patient responsiveness and pathway set-up.

\section{Optimised scenarios}

Additional scenarios were defined to interpret findings on the effects of the implementation of best practices (or combinations thereof) by generating upper boundaries to pathway performance, thereby building on unrealistically optimistic assumptions. For the optimised scenarios, we extrapolated best practices by setting the parameter for associated time delays to 0 or by setting the diagnostic quality parameter to $100 \%$.

\section{Outcome measures}

The primary outcome measure was the proportion of patients treated with IVT. Secondary outcome measures were the total process time (onset-to-treatment time), IVT within various time intervals (0-90, 91-180 and 181-270 min), the proportion of patients with favourable outcomes at 90 days (mRS $0-1$ ) and additional healthy life days (calculated using OTT estimates). ${ }^{12} 35$

\section{Analysis}

For each of the scenarios described above, we calculated new hypothetical IVT treatment rates and secondary outcome measures, based on the number of patients arriving in time for acute treatment at the hospital (ie, within 4 hours after symptom onset), the number treated with IVT and the time to treatment. $\chi^{2}$ tests were used to compare categorical variables.

\section{Ethics approval and patient consent}

Informed consent was obtained from all subjects participating in the prospective study ${ }^{9}$ and extended for the current simulation study.

\section{Patient and public involvement}

Patients and public were not involved in the design of this study. For this modelling study non-identifiable patient data was used. Study results will be disseminated through poster presentations and publication in peer-reviewed journals.

\section{RESULTS}

This study reflects the experiences of 280 ischaemic stroke patients referred to the UMCG and three community hospitals, as part of a centralised organisational model. Baseline and demographic characteristics are described in table 1. In all, $125(44.6 \%)$ patients arrived at the hospital within 4.5 hours, $61(21.8 \%)$ received IVT, and the median OTT was $127 \mathrm{~min}$. Of the patients receiving IVT, $17.0 \%$ were treated within $90 \mathrm{~min}$ of symptom onset. Patient delay, intrahospital delays and mode of referral (GP or 911) were identified as the greatest obstacles to receiving IVT (table 1 ).

\section{Simulation experiments}

The results of the simulation experiments are presented in table 3.

Patient responsiveness: If patients had contacted emergency services sooner (ie, within 30 and $15 \mathrm{~min}$ ), up to $27.6 \%$ (CI 26.7\% to 28.4\%) of the total population would have been treated with IVT (table 3, Scenarios 1A and $1 \mathrm{~B})$, and the OTT would have been reduced to $122 \mathrm{~min}$ (CI 121 to 124). ${ }^{27} 28$

Assuming a patient delay of $0 \mathrm{~min}$ (table 3, Scenario 1C), $64.0 \%$ of the total population would have been treated with IVT, and the OTT would have been reduced to $92 \mathrm{~min}$ (CI 91 to 92 ).

Mode of referral: Assuming $60 \%$ of all patients contacting 911 immediately following stroke onset (table 3, Scenario 2A) increased the IVT rate to $24.6 \%$ (CI $23.8 \%$ to $25.5 \%$ ) and reduced the OTT to $127 \mathrm{~min}$ (CI 125 to 128 ). ${ }^{29}$

If all patients $(100 \%)$ had called 911 (table 3, Scenario 2B), the IVT rates would have increased further to $28.4 \%$ (CI $27.5 \%$ to $29.3 \%$ ), and the OTT would have been reduced to $124 \mathrm{~min}$ (CI 123 to 126 ).

Time spent on the scene by ambulance personnel: Shortening the time spent on the scene to 15 and $10 \mathrm{~min}$ (table 3, Scenarios $6 \mathrm{~A}$ and $6 \mathrm{~B}),{ }^{36}$ increased the IVT treatment rate up to $23.3 \%$ (CI $22.4 \%$ to $24.1 \%$ ) and reduced the OTT to $121 \mathrm{~min}$ (CI 120 to 123 ). ${ }^{3738}$

Reducing time on the scene to 0 min resulted in a projected IVT rate of $24.7 \%$ (CI 23.8\% to $25.5 \%$ ) (table 3 , Scenario 6C) and a decrease in the OTT to $114 \mathrm{~min}$ (CI 112 to 116 ).

Prehospital treatment by MSU: In this set-up, 23.2\% (CI $22.4 \%$ to $24.0 \%$ ) of patients would have been treated with IVT (table 3, Scenario 7A) and OTT would have been reduced to $121 \mathrm{~min}$ (CI 120 to 123 ).

The elimination of both the response time and transportation time of the MSU (table 3, Scenario 7B) resulted in a projected $25.6 \%$ (CI $24.7 \%$ to $26.4 \%$ ) of patients that could have received IVT and a reduction of the OTT to 109 min (CI 107 to 110 ).

Intrahospital processes: If the DTN time had been shortened to a maximum of 30 and $20 \mathrm{~min}$ (table 3, Scenarios $8 \mathrm{~A}-\mathrm{C}$ ), up to $26.6 \%$ (CI $25.7 \%$ to $27.4 \%$ ) of the total population would have been treated with IVT, and the OTT would have been reduced to $101 \mathrm{~min}$ (CI 99 to 103). 


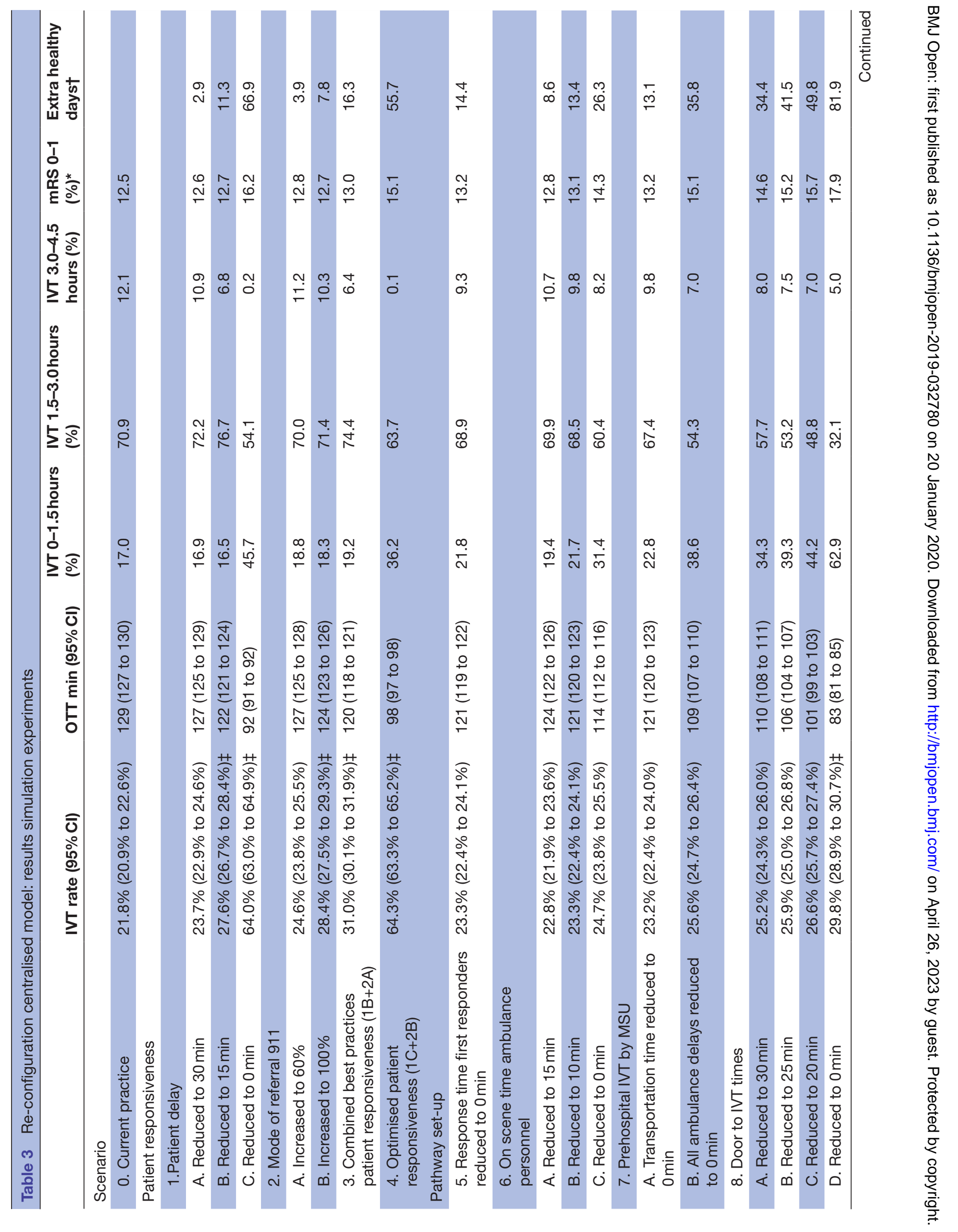




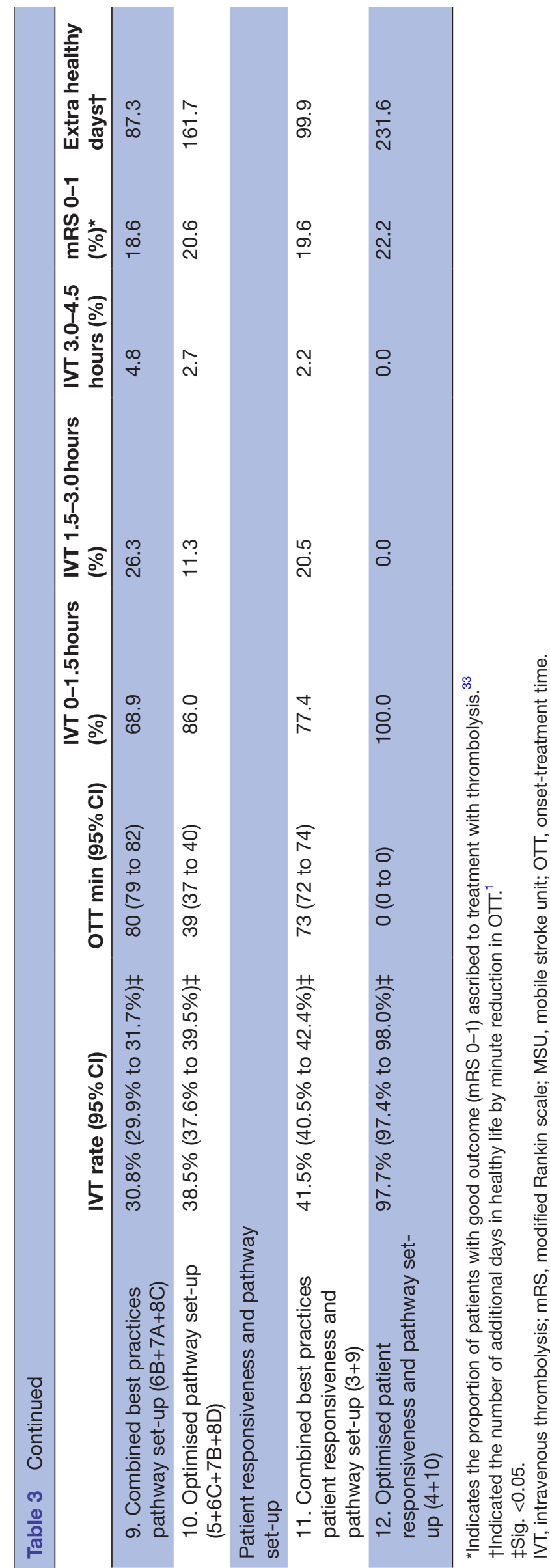

If the DTN had been reduced to $0 \mathrm{~min}$ (table 3 , Scenario $8 \mathrm{D}$ ), $29.8 \%$ (CI $28.9 \%$ to $30.7 \%$ ) of all patients would have been treated with IVT, and the OTT would have been reduced to $83 \mathrm{~min}$ (CI 81 to 85 ).

\section{Combined best practice scenarios}

Combining best practices for patient responsiveness and pathway set-up (table 3 , scenario's $3,9,11$ ) resulted in up to $41.5 \%$ (CI $40.5 \%$ to $42.4 \%$ ) of all patients being treated with IVT and reduced the OTT to $73 \mathrm{~min}$ (CI 72 to 74 ).

\section{Optimised scenarios}

Assuming optimised patient responsiveness (ie, all patients calling 911 immediately following stroke onset; table 3, Scenario 4) resulted in $64.3 \%$ of the total population (CI $63.3 \%$ to $65.2 \%$ ) being treated with IVT and reduced the OTT to $98 \mathrm{~min}$ (CI 97 to 98 ). The optimisation of pathway set-up (table 3, Scenario 10) resulted in $38.5 \%$ (CI $37.6 \%$ to $39.5 \%)$ of all patients receiving IVT and reduced the OTT to $39 \mathrm{~min}$ (CI 37 to 40 ). The combination of all optimised scenarios (table 3, Scenario 12) resulted in a cumulative total of $97.7 \%$ (CI $97.4 \%$ to $98.0 \%$ ) of all patients being treated with IVT and reduced the OTT to $0 \mathrm{~min}$.

\section{DISCUSSION}

This study demonstrates that IVT treatment rates above $30 \%$ would be possible if best practices were to be implemented within our setting. We modelled several scenarios to generate insight into the potential for quality improvements in our acute stroke chain of care. Although improvements in patient responsiveness would yield the largest potential gains within our pathway, even modest changes in this regard are likely to be challenging and costly to achieve. ${ }^{16}$ In contrast, improvements in other areas (eg, intrahospital delays and time spent by ambulance personnel at the patient's location) might be easier to achieve and would still lead to clinically relevant increases in IVT rates. As indicated in previous studies, even small reductions in time to treatment with IVT are associated with considerable increases in the length of healthy life, and they may require only relatively simple organisational changes involving minimal effort at little or no cost. ${ }^{12}$

The results of our study may be useful as a guide for prioritising interventions along the acute stroke pathway and for estimating their potential impact on the effectiveness of the pathway. A simulation-based approach, as presented in this paper, can be instrumental in facilitating a broad overview of the set-up and performance of stroke pathways. This could provide clinicians and policymakers with speedy answers-at little effort or costconcerning how new or widely advocated practices could be used to improve their pathways, thus allowing them to direct investments to the interventions that matter most. It thus has the potential to replace RCT studies or serve as a precursor to a focussed RCT, which could be scoped as the net result of a simulation approach. 
In our study, we observed the greatest effects on IVT rates, time to treatment and patient outcomes after improving the responsiveness of patients and/or bystanders by reducing the time from symptom onset to the call for help, thereby expediting intrahospital care services and by increasing the number of 911 calls made by patients or bystanders. In contrast, a scenario in which prehospital transportation delays were reduced by the implementation of a MSU resulted in only moderate effects. Combining all of the best-practice scenarios resulted in a maximum of $41.5 \%$ of patients being treated with IVT. This is substantially higher than current benchmark figures on clinical practice, which suggest a maximum IVT rate of around $35 \% .^{639}$

Proceeding from a scenario in which best practices have been implemented, remaining challenges include realising further decreases in time delays in both the prehospital and intrahospital phases. The feasibility of such initiatives in clinical practice might be limited in the short term, however, given the current lack of evidence concerning solutions for further expediting care and logistics services at reasonable costs. For example, the goal of reducing time spent on the scene by ambulance personnel to less than 10 min or reducing DTN time to less than $20 \mathrm{~min}$ would probably be unrealistic, given the need to handle and observe the patient, to complete diagnostic tests and to interpret findings. Although further improvements in the proportion of patients calling 911 directly following symptom onset could potentially result in further increases in IVT rates, they would also necessitate large-scale and repetitive publicity campaigns comparable to those launched to raise public awareness on stroke symptoms and how to act.

The organisational model for acute stroke care delivery is currently receiving a great deal of attention in the Netherlands, as well as beyond. ${ }^{40}$ The emergence of endovascular treatment (EVT) for patients facing largevessel occlusions has opened up a whole new dimension in terms of acute stroke pathway set-up and patient logistics. Following IVT, eligible patients must now undergo additional diagnostic evaluation (eg, CT angiography and perfusion CT), followed by such EVT treatment modalities as groin punctures and initial attempt at clot retrieval with the device up to the angioseal following successful recanalisation. In addition, within the current 'drip-and-ship' treatment paradigm, eligible patients may initially be admitted to community hospitals before being transferred to comprehensive stroke centres with EVT capacity, thereby further increasing the number of logistical steps. Given the time-sensitive nature of acute stroke interventions, this extension of the pathway necessitates the re-organisation of acute stroke care within regions and settings. In this respect, simulation modelling could facilitate insight into the complex interplay of separate elements of the pathway. Currently positioned as a follow-up treatment by current guidelines, availability of EVT does not change the need for optimising utilisation of IVT, nor does it impact the acute stroke pathway set-up for IVT. Moreover, the subgroup of patients eligible for EVT is relatively small, around $7 \%$ of all stroke patients. ${ }^{42}$

Our study is subject to several limitations. First, our simulation models did not consider the response of GPs when contacted as first responders. Although this has been signalled as an issue for delays in hospital arrival for patients, ${ }^{43}$ no studies on best practices were identified in the literature. Second, because the costs and cost-effectiveness associated with pathway improvements in our setting were not estimated in our model, it was not possible to control for them. Third, the results of our findings might not be generalisable to other settings, due to the unique position of the UMCG, which serves as a stroke centre in a centralised organisational model deployed within its region. As noted in a previous publication, however, the generic modelling approach adopted in this study could be extended to include a description of a decentralised organisational model, which receives IVT candidates within its own catchment areas. ${ }^{23}$ Also, because patients were enrolled in the observational study back in 2010 these results will not fully reflect current practice. However, review of internal databases show that IVT treatment percentages have remained largely stable over the last years, fluctuating around 25\% with a DTN time of around $35 \mathrm{~min}$. EMS response times have remained constant over the years. ${ }^{44}$ In addition, pathway set-up of acute stroke patients receiving IVT remained similar over the years. Finally, model assumptions excluded the possibility for capacity constraints influencing patient waiting times, as patients and/or transport queuing seldom occurs due to the high prioritisation that potential acute stroke patients receive throughout the pathway. However, we acknowledge that such constraints might occur in other stroke care systems. Future activities should be aimed at extending the simulation-based approach to include the drip-and-ship model currently employed in acute stroke treatment (eg, EVT).

\section{CONCLUSIONS}

The results of this study indicate that the cumulative effects of implementing best practices on the organisation of stroke care would clearly exceed current benchmarks for treatment rates. Remaining obstacles might be difficult to overcome given the limited availability of solution to further expedite care and logistical services at tolerable costs. A broader overview facilitated by simulation is suggested as instrumental in supporting decision-makers and clinicians in their efforts to evaluate the set-up and performance of acute stroke pathways.

Contributors MMHL and D-JZ designed the study, performed the experiments and analysed the data. MMHL drafted the manuscript, D-JZ, G-JL and EB critically revised the manuscript for intellectual content and approved the final version of the manuscript for publication.

Funding The authors have not declared a specific grant for this research from any funding agency in the public, commercial or not-for-profit sectors.

Competing interests None declared.

Patient consent for publication Not required. 
Ethics approval No additional approval was necessary because for this simulation study an anonymised dataset was used.

Provenance and peer review Not commissioned; externally peer reviewed.

Data availability statement Data are available upon reasonable request.

Open access This is an open access article distributed in accordance with the Creative Commons Attribution 4.0 Unported (CC BY 4.0) license, which permits others to copy, redistribute, remix, transform and build upon this work for any purpose, provided the original work is properly cited, a link to the licence is given, and indication of whether changes were made. See: https://creativecommons.org/ licenses/by/4.0/.

ORCID iD

Maarten M H Lahr http://orcid.org/0000-0001-7265-2612

\section{REFERENCES}

1 The National Institute of neurological disorders and stroke rt-PA stroke Study Group. Tissue plasminogen activator for acute ischemic stroke. N Engl J Med Overseas Ed 1995;333:1581-8.

2 Hacke W, Kaste M, Bluhmki E, et al. Thrombolysis with alteplase 3 to 4.5 hours after acute ischemic stroke. N Engl J Med 2008;359:1317-29.

3 Bauer A, Limburg M, Visser MC. Variation in clinical practice of intravenous thrombolysis in stroke in the Netherlands. Cerebrovasc Dis Extra 2013;3:74-7.

4 Adeoye $\mathrm{O}$, Hornung $\mathrm{R}$, Khatri $\mathrm{P}$, et al. Recombinant tissue-type plasminogen activator use for ischemic stroke in the United States: a doubling of treatment rates over the course of 5 years. Stroke 2011;42:1952-5

5 Waite K, Silver F, Jaigobin C, et al. Telestroke: a multi-site, emergency-based telemedicine service in Ontario. J Telemed Telecare 2006;12:141-5.

6 Stolz E, Hamann GF, Kaps M, et al. Regional differences in acute stroke admission and thrombolysis rates in the German federal state of Hesse. Dtsch Arztebl Int 2011;108:607-11.

7 Bekelis K, Marth NJ, Wong K, et al. Primary stroke center hospitalization for elderly patients with stroke: implications for case fatality and travel times. JAMA Intern Med 2016;176:1361-8.

8 Willeit J, Geley T, Schöch J, et al. Thrombolysis and clinical outcome in patients with stroke after implementation of the Tyrol stroke pathway: a retrospective observational study. Lancet Neurol 2015;14:48-56.

9 Lahr MMH, Luijckx G-J, Vroomen PCAJ, et al. Proportion of patients treated with thrombolysis in a centralized versus a decentralized acute stroke care setting. Stroke 2012;43:1336-40.

10 Ramsay AIG, Morris S, Hoffman A, et al. Effects of centralizing acute stroke services on stroke care provision in two large metropolitan areas in England. Stroke 2015;46:2244-51.

11 Morris S, Hunter RM, Ramsay AIG, et al. Impact of centralising acute stroke services in English metropolitan areas on mortality and length of hospital stay: difference-in-differences analysis. BMJ 2014;349:g4757.

12 Meretoja A, Keshtkaran M, Saver JL, et al. Stroke thrombolysis: save a minute, save a day. Stroke 2014;45:1053-8.

13 Tsivgoulis G, Katsanos AH, Kadlecová P, et al. Intravenous thrombolysis for ischemic stroke in the golden hour: propensitymatched analysis from the SITS-EAST registry. $J$ Neurol 2017;264:912-20.

14 Kleindorfer D, Khoury J, Broderick JP, et al. Temporal trends in public awareness of stroke: warning signs, risk factors, and treatment. Stroke 2009;40:2502-6.

15 Fassbender K, Balucani C, Walter S, et al. Streamlining of prehospital stroke management: the golden hour. Lancet Neurol 2013;12:585-96.

16 Wakefield MA, Loken B, Hornik RC. Use of mass media campaigns to change health behaviour. The Lancet 2010;376:1261-71.

17 Evenson KR, Foraker RE, Morris DL, et al. A comprehensive review of prehospital and in-hospital delay times in acute stroke care. Int $J$ Stroke 2009;4:187-99.

18 Rose KM, Rosamond WD, Huston SL, et al. Predictors of time from hospital arrival to initial brain-imaging among suspected stroke patients: the North Carolina collaborative stroke Registry. Stroke 2008;39:3262-7.

19 Fassbender K, Grotta JC, Walter S, et al. Mobile stroke units for prehospital thrombolysis, triage, and beyond: benefits and challenges. Lancet Neurol 2017;16:227-37.
20 Meretoja A, Strbian D, Mustanoja S, et al. Reducing in-hospital delay to 20 minutes in stroke thrombolysis. Neurology 2012;79:306-13.

21 Lahr MMH, van der Zee D-J, Vroomen PCAJ, et al. Thrombolysis in acute ischemic stroke: a simulation study to improve pre- and inhospital delays in community hospitals. PLoS One 2013;8:e79049.

22 Monks T, Pitt M, Stein K, et al. Maximizing the population benefit from thrombolysis in acute ischemic stroke: a modeling study of inhospital delays. Stroke 2012;43:2706-11.

23 Lahr MMH, van der Zee D-J, Luijckx G-J, et al. Centralising and optimising decentralised stroke care systems: a simulation study on short-term costs and effects. BMC Med Res Methodol 2017;17:016-275.

24 Lahr MMH, van der Zee D-J, Luijckx G-J, et al. A simulation-based approach for improving utilization of thrombolysis in acute brain infarction. Med Care 2013;51:1101-5.

25 Wahlgren N, Ahmed N, Dávalos A, et al. Thrombolysis with alteplase for acute ischaemic stroke in the safe implementation of thrombolysis in Stroke-Monitoring study (SITS-MOST): an observational study. The Lancet 2007;369:275-82.

26 Law AM. ExpertFit version 8 user's guide. Tuscon, Arizona: Averill M. Law \& Associates, 2011

27 Salisbury Het al. Delay in presentation of patients with acute stroke to hospital in Oxford. QJM 1998;91:635-40.

28 Carroll C, Hobart J, Fox C, et al. Stroke in Devon: knowledge was good, but action was poor. J Neurol Neurosurg Psychiatry 2004;75:567-71.

29 Barsan WG, Brott TG, Broderick JP, et al. Urgent therapy for acute stroke. Effects of a stroke trial on untreated patients. Stroke 1994;25:2132-7.

30 Chen N-C, Hsieh M-J, Tang S-C, et al. Factors associated with use of emergency medical services in patients with acute stroke. Am J Emerg Med 2013;31:788-91.

31 Powers WJ, Rabinstein AA, Ackerson T, et al. 2018 guidelines for the early management of patients with acute ischemic stroke: a guideline for healthcare professionals from the American heart Association/ American stroke association. Stroke 2018;49:e46-110.

32 Fonarow GC, Smith EE, Saver JL, et al. Timeliness of tissuetype plasminogen activator therapy in acute ischemic stroke: patient characteristics, hospital factors, and outcomes associated with door-to-needle times within 60 minutes. Circulation 2011;123:750-8.

33 Zinkstok SM, Beenen LF, Luitse JS, et al. Thrombolysis in stroke within 30 minutes: results of the acute brain care intervention study. PLoS One 2016;11:e0166668.

34 Meretoja A, Weir L, Ugalde M, et al. Helsinki model cut stroke thrombolysis delays to 25 minutes in Melbourne in only 4 months. Neurology 2013;81:1071-6.

35 Lees KR, Bluhmki E, von Kummer R, et al. Time to treatment with intravenous alteplase and outcome in stroke: an updated pooled analysis of ECASS, atlantis, NINDS, and epithet trials. The Lancet 2010;375:1695-703.

36 Atkins DL, Everson-Stewart S, Sears GK, et al. Epidemiology and outcomes from out-of-hospital cardiac arrest in children: the resuscitation outcomes Consortium Epistry-Cardiac arrest. Circulation 2009;119:1484-91.

37 Jauch EC, Saver JL, Adams HP, et al. Guidelines for the early management of patients with acute ischemic stroke: a guideline for healthcare professionals from the American heart Association/ American stroke association. Stroke 2013;44:870-947.

38 Acker JE, Pancioli AM, Crocco TJ, et al. Implementation strategies for emergency medical services within stroke systems of care: a policy statement from the American heart Association/American stroke association expert panel on emergency medical services systems and the stroke Council. Stroke 2007;38:3097-115.

39 Boode B, Welzen V, Franke C, et al. Estimating the number of stroke patients eligible for thrombolytic treatment if delay could be avoided. Cerebrovasc Dis 2007;23:294-8.

40 Lahr MMH, van der Zee D-J, Luijckx G-J, et al. Improving acute stroke services in the Netherlands. BMJ 2014;348:g3957.

41 Monks T, Pitt M, Stein K, et al. Hyperacute stroke care and NHS England's business plan. BMJ 2014;348:g3049.

42 Chia NH, Leyden JM, Newbury J, et al. Determining the number of ischemic strokes potentially eligible for endovascular thrombectomy. Stroke 2016;47:1377-80.

43 Caminiti C, Schulz P, Marcomini B, et al. Development of an education campaign to reduce delays in pre-hospital response to stroke. BMC Emerg Med 2017;17:017-130.

44 Table book 2018. Emergency care Netherlands. Zwolle, 2019. 